\title{
Germanica
}

\section{„Ein großartiger Entertainer !“ Kritik als Unterhaltung oder Unterhaltung als Kritik bei Marcel Reich-Ranicki}

Caspar BATTEGAY : "Un maître du divertissement »! La critique comme divertissement ou le divertissement comme critique chez Marcel Reich-Ranicki Caspar BATTEGAY: "A Great Entertainer!" Critique as Entertainment and Entertainment as Critique

\section{Caspar Battegay}

\section{OpenEdition}

Journals

Édition électronique

URL : https://journals.openedition.org/germanica/7425

DOI : $10.4000 /$ germanica. 7425

ISSN : 2107-0784

\section{Éditeur}

Université de Lille

\section{Édition imprimée}

Date de publication : 1 décembre 2019

Pagination : 31-46

ISBN : 978-2-913857-44-5

ISSN : 0984-2632

\section{Référence électronique}

Caspar Battegay, " Ein großartiger Entertainer !" Kritik als Unterhaltung oder Unterhaltung als Kritik bei Marcel Reich-Ranicki“, Germanica [Online], 65 | 2019, Online erschienen am: 01 Januar 2022, abgerufen am 08 Januar 2022. URL: http://journals.openedition.org/germanica/7425 ; DOI: https://doi.org/ $10.4000 /$ germanica.7425 


\title{
„Ein großartiger Entertainer!“ Kritik als Unterhaltung oder Unterhaltung als Kritik bei Marcel Reich-Ranicki
}

\author{
Caspar Battegay \\ Universität Basel
}

\section{Unterhaltung und Konsumästhetik}

$\mathrm{Da}$ der Mensch unterhalten sein will, ist noch immer für den Menschen der stärkste Antrieb, sich mit den Produkten der Schriftstellerei zu beschäftigen; indem sie den menschlichen Unterhaltungstrieb einkalkulieren, schreiben gerade große Schriftsteller oft amüsant, sie verstehen ihr Geschäft ${ }^{1}$.

Diese Sentenz Friedrich Dürrenmatts stammt aus einem Vortrag von 1956. Marcel Reich-Ranicki zitiert sie 1966 in der ZEIT anlässlich des Erscheinens des ersten Bandes von Dürrenmatts gesammelten TheaterSchriften und Reden. Reich-Ranickis Artikel stellt eine grundsätzliche Würdigung des Schweizer Dramatikers, Essayisten und Prosaautors dar ${ }^{2}$. Zudem enthält er nicht allzu versteckt ein kleines Selbstporträt

1. - Friedrich Dürrenmatt, „Schriftstellerei als Beruf“, in: Ders., TheaterSchriften und Reden, Zürich, Verlag der Arche, 1966, S. 50-56, hier S. 56.

2. - Marcel Reich-Ranicki, ,Verbeugung vor einem Raubtier. Die Theaterschriften 
des Kritikers selbst: „Häufig wird ihm - wenn auch mitunter halb widerwillig - bescheinigt, was man schwerlich leugnen kann: Humor, Intuition, Originalität, Einfallsreichtum, handwerkliche Meisterschaft. Aber man billigt ihm eher Phantasie als Geist zu, eher Schlagfertigkeit als Tiefe, eher Esprit als Weisheit"3.

Dieses ,halb widerwillige' Bescheinigen von Schlagfertigkeit und ,Witz' zuungunsten der ,Tiefe' findet sich beispielsweise in Franz Josef Czernins Essay Marcel Reich-Ranicki. Eine Kritik (1995). Czernin kommt in dieser gut 200-seitigen Metakritik ebenfalls auf ReichRanickis oben genanntes Dürrenmatt-Zitat zu sprechen - als ein Beweis für Reich-Ranickis zwar begeistertes, aber vermeintlich oberflächliches und populistisches Verständnis von Literatur ${ }^{4}$. Denn für Czernin impliziert Dürrenmatts Hinweis auf den „Unterhaltungstrieb“ und das marktgängige „Geschäft" der Literatur eine unreflektierte Bevorzugung des literarischen ,Handwerks' zuungunsten eines diffusen Begriffs der künstlerischen, Avantgarde'. Diese würde Reich-Ranicki als Fetischisierung des letztlich unverständlichen Textes diffamieren. Gemäß Czernin habe dieser rhetorisch geschickt getarnte Antimodernismus literaturhistorische Dimensionen; er knüpfe an einen „Realismusbegriff“ an, „der im deutschen Sprachraum seit ungefähr 1840 eine der maßgeblichen Kräfte darstellt“ und der mit einer „Kritik an der Bezugslosigkeit der Romantiker zu einer angeblich historisch objektiven Wirklichkeit" 5 verbunden sei. Diese Reich-Ranicki unterschobene Ansicht basiert auf einem Verständnis von Literatur, das den Konstruktionscharakter jeder textuellen Wirklichkeitsreferenz ignoriere.

Czernins hier nur sehr verkürzt wiedergegebene Argumentation soll in diesem Aufsatz nicht angezweifelt werden. Tatsächlich hat ReichRanickis Konzentration auf den Stoff und das Geschichtenerzählen eine reduktionistische Tendenz $z^{6}$. Allerdings ist dies auch in Czernins abwertendem Verständnis des Markts und der Unterhaltungsfunktion sowie seines empathischen Begriffs einer autonomen und (sowohl formal wie

und Reden Friedrich Dürrenmatts“, DIE ZEIT, 44/1966, https://www.zeit.de/1966/44/ verbeugung-vor-einem-raubtier (Zugriff am 05.06.2019).

3. - Ebd.

4. - Franz Joseph Czernin, Marcel Reich-Ranicki. Eine Kritik, Göttingen, Steidl, 1995, S. 30.

5. - Ebd., S. 16.

6. - Ein beliebiges Beispiel wäre, dass Reich-Ranicki den Schriftsteller Joseph Roth primär als ,ein[en] elementare[n], ein[en] spontane[n] Geschichtenerzähler“ einschätzt - und Roths reflektierten Modernismus, die sorgfältige Konstruiertheit der Texte vollkommen ignoriert und so letztlich auch die Textualität und Literarizität von Roths Literatur nicht erkennt. Marcel Reich-Ranicki, ,Joseph Roth, der Romancier“, in: Michael Kessler/ Fritz Hackert (Hrsg.), Joseph Roth. Interpretation - Rezeption - Kritik. Akten des internationalen, interdisziplinären Symposions 1989, Akademie der Diözese Rottenburg-Stuttgart, Tübingen, Stauffenburg Verlag, 1990, S. 261-268, hier S. 265. 
inhaltlich) widerständigen Kunst der Fall. Dies zeigt sich schon darin, dass Czernin sich nicht die Mühe macht, Dürrenmatts Zitat im Original nachzulesen. Dort zeigt sich nämlich ein wichtiges, die Komplexität stark erhöhendes Detail: Vor dem zitierten Absatz, der den Schluss und damit auch eine Art Pointe von Dürrenmatts Text bildet, steht noch das Wort „Trost:“" mit einem Doppelpunkt. Die Sentenz formuliert also keineswegs ein Ideal. In seinem Vortragstext vergleicht Dürrenmatt die gesellschaftliche Position des Schriftstellers in der Sowjetunion mit dem vermeintlich freien Westen. Er kommt zum Schluss, dass es mit der Autonomie des Künstlers auch im Westen nicht weit her sei, weil er sich nach den Bedürfnissen des Marktes richten und „listig schreiben“7 müsse, um seine Existenz im Kapitalismus zu sichern. Das anthropologische Bedürfnis der Unterhaltung stelle also einen „Trost“ dar, mit dem ein Schriftsteller sich in einer ansonsten nutzen- und konsumorientierten Gesellschaft pragmatisch einrichten könne. Damit knüpft Dürrenmatt an die klassische Begriffsgeschichte der Unterhaltung als delectatio an: Gerade in der ,zweckfreien Natur der U.[nterhaltung] “ wird öfters „,das Potential zu Kreativität und affektiver Wirkungskraft" 8 gesehen, was letztlich auf das berühmte Horaz'sche Diktum aut prodesse volunt aut delectare poetae zurückgeht.

In diesem Beitrag zeige ich, dass der Begriff der Unterhaltung keineswegs jene Eindimensionalität aufweist, die ihm in ästhetischen Debatten oft zugesprochen wird. Vielmehr verweisen die cultural studies darauf $^{9}$, dass moderne und populäre Unterhaltung ein vielschichtiges Phänomen ist, das die Möglichkeit zur kreativen Reflexion und zur Selbstreflexion einschließt sowie Ironie und Ambiguität durchaus zulässt, wenn nicht sogar provoziert.

Die kritische Theorie versteht Unterhaltung im Kontext der marktwirtschaftlichen Mediengesellschaft als schematisch organisiertes ,Amüsement'. In dieser Sicht ist Unterhaltung ein Teil der ,Kulturindustrie', der das Publikum von ihrem totalitären ,Verblendungszusammenhang ${ }^{\circ}$ ablenkt. Dieser eher denunzierende als deskriptive Ansatz entspricht kaum mehr der Erfahrung unserer Lebenswelt, in der wir - wie jüngst Moritz Baßler und Heinz Drügh festhielten - längst auch Konsumgüter und Produkte der Unterhaltungsindustrie ästhetisch differenziert beurteilen beziehungsweise eben nicht bloß ihren Gebrauchswert wahrnehmen, sondern die Produkte und Marken mit vielfältigen kulturellen Konnotationen versehen. Aber nicht nur auf der

7. — F. Dürrenmatt, ,Schriftstellerei als Beruf“, a.a.O., S. 56.

8. - Thomas Pekar, „Unterhaltung“, in: Historisches Wörterbuch der Rhetorik, hrsg. von Gerd Ueding, Bd. 9, Tübingen, Niemeyer, 1992, Sp. 914-932, hier Sp. 915.

9. - Vgl. Kerstin Goldbeck, Gute Unterhaltung, schlechte Unterhaltung. Die Fernsehkritik und das Populäre, Bielefeld, transcript, 2004, S. 13-22. 
Seite der Konsumierenden, sondern auch auf der Angebotsseite sind Marktstrategien, Werbung, Produktdesign etc. an das ironische Spiel des Konsums angepasst. Im Kontext einer solchen „Konsumästhetik“ kann Unterhaltung „,nicht in gängiger konsumkritischer Manier als das uniforme und unachtsame, Sich-Reinziehen' von unterschiedslos Allem bewertet, sondern als hochkomplexe Handlungsform" 10 gesehen werden. Unsere Beziehung zu Konsumgütern wird von vielfältigen Emotionen und Imaginationen begleitet, mit denen wir zu einem großen Teil auch bewusst umgehen.

Marcel Reich-Ranicki, dessen Image als „Popstar der Kritik“11 zu einem beträchtlichen Teil auf dessen Unterhaltsamkeit zurückzuführen ist, dessen Unterhaltsamkeit aber umgekehrt auch auf gerade dieses markenäquivalente Image, sprengt meines Erachtens in genau dieser von Baßler und Drügh beschriebenen Weise die romantisch aufgeladene Kunst- und Werkästhetik ${ }^{12}$. Er führt vor, wie über Literatur unter den Bedingungen ihrer Konsumierbarkeit und des Literaturmarktes gesprochen werden kann, so dass ihr kreatives und affektives Potential ironisch hervortritt, und so, dass das literarische Pathos der Unmittelbarkeit in seiner oftmals reaktionären oder zumindest anti-liberalen Funktion neutralisiert wird.

Mein Beitrag soll also erörtern, inwiefern Marcel Reich-Ranicki als Autor, Performer und Figur sich in seiner Kritik nicht nur der Unterhaltung bedient, sondern als Unterhalter auch ein ästhetischkritisches Potential besitzt, das sich nicht gegen, sondern im performativen Umgang mit dem Unterhaltungsbusiness entfaltet. Dieses Potential werde ich nun unter drei Aspekten diskutieren: erstens hinsichtlich von Literatur und Literaturkritik im Text; zweitens hinsichtlich des Mediums Fernsehen; und drittens hinsichtlich der Repräsentation des Holocausts, also der Wahrnehmung Reich-Ranickis als jüdischer Überlebender.

\section{Literatur/Kritik als Unterhaltung}

In seiner Autobiografie Mein Leben (1999) schildert Marcel Reich-Ranicki anhand einer prägenden Lehrerfigur ein literarisches Initiationserlebnis. Sein Deutschlehrer Reinhold Knick habe den ersten Akt von Gerhard

10. - Moritz Baßler/ Heinz Drügh, „Einleitung: Konsumästhetik“, in: Dies. (Hrsg.), Konsumästhetik. Umgang mit käuflichen Gegenständen, Bielefeld, transcript, 2019, S. 7-26, hier S. 9-10.

11. - Uwe Wittstock, Marcel Reich-Ranicki. Die Biographie, München, Karl Blessing, 2015, S. 12.

12. - Aus soziologischer Perspektive wurde das Image auch mit Pierre Bourdieus Begriff der posture zu erfassen versucht, vgl. Jasmin Ahmadi, Der Papst und der Bienenkorb: Marcel Reich-Ranicki als ein Akteur im literarischen Feld der Bundesrepublik, Frankfurt a.M., Academic Research Peter Lang, 2015, S. 249-261. 
Hauptmanns Komödie Der Biberpelz vorgelesen, was den jungen Marcel dazu veranlasst hätte, sich ,gleich etwa ein halbes Dutzend der Dramen Hauptmanns“13 zu Gemüte zu führen. „Ich begriff, dass Literatur unterhaltsam sein darf - und sein sollte. Ich habe es nie vergessen"14.

Der Kritiker kommt in seinen Aufsätzen und Äußerungen tatsächlich immer wieder auf das „Unterhaltungsbedürfnis“ 15 als Kern der Literatur zurück und fordert von der Literatur denn auch Unterhaltung. „Unterhaltungsprosa“ definiert er bereits 1962 als „Romane, deren Autoren es sich sehr schwer machen, damit die Leser es sehr leicht haben; Unterhaltungsromane also, die harmlos und anspruchslos sein dürfen, ja sein sollen, zugleich jedoch logisch, vernünftig und intelligent, geschmacksvoll und spannend, geistreich und humorvoll sind"16. Er konstatiert, dass außer einigen bemerkenswerten Ausnahmen eine solche Mischung in der deutschen Literatur kaum existiere, vielmehr tue sich ,ein Niemandsland zwischen Kolportage und großer Kunst"17 auf. Die Opposition von Trivialität und Avantgarde wird jedoch gerade durch den Begriff der Unterhaltung brüchig - und die von Reich-Ranicki genannten Autoren wie Theodor Fontane, Arthur Schnitzler, Erich Kästner oder Friedrich Dürrenmatt, die jene schmale Linie der deutschsprachigen Unterhaltungsliteratur begründen würden, bestätigen, dass komplexe Ästhetik und Unterhaltung sich auch bedingen können.

Als Kritiker und Autor stellt sich Reich-Ranicki selbst in jene Genealogie des Klaren und Nüchternen, die er der „Dämmerung“ und der „Beschwörung“ entgegensetzt ${ }^{18}$. Er selbst stilisiert sich in recht simpler Weise als Bewohner des konstatierten ,Niemandslandes' und als Störfaktor, der im Sinn der Aufklärung zu einem gesellschaftlichen Dialog beitrage. Doch die von ihm des Öfteren eingeforderte „maximale Deutlichkeit"19 der Kritik geht auch mit Unterhaltsamkeit einher. Diese kritische Unterhaltsamkeit verdankt sich vielleicht auch der Lehrerfigur Reinhold Knicks, der in Mein Leben als solidarisch

13. - Marcel Reich-Ranicki, Mein Leben, Deutscher Taschenbuch Verlag, München, 2. Auflage, 2001 [2000], S. 50.

14. - Ebd.

15. - So beispielsweise 1986 im Gespräch mit Peter von Matt, in dem sich ReichRanicki auf eine entsprechende Äußerung Bertolt Brechts bezieht. Siehe Marcel ReichRanicki, Der doppelte Boden. Ein Gespräch mit Peter von Matt, Zürich, Ammann, 1992, S. 38. Eine zentrale Bemerkung Reich-Ranickis ist in diesem Zusammenhang: „Ich pfeife auf die Wahrheit in der Literatur und die in ihr dargestellte Philosophie, wenn mich diese Literatur langweilt." S. 63.

16. - Marcel Reich-Ranicki, „Deutsche Unterhaltungsliteratur“, in: Ders., Nichts als Literatur. Aufsätze und Anmerkungen, Stuttgart, Reclam, 1985, S. 14-17, hier S. 17.

17. - Ebd.

18. - Vgl. Marcel Reich-Ranicki, Über Literaturkritik, Stuttgart/München, Deutsche Verlags-Anstalt, 2002 (2. Auflage), S. 42.

19. — Ebd. S. 59. 
mit seinen jüdischen Schülern geschildert wird, als später Vertreter des deutschen Idealismus ${ }^{20}$. Doch die Unterhaltungsfunktion gehört als eine von verschiedenen anderen Funktionen (Information, Auswahl, didaktische Vermittlung, didaktische Sanktionen, Reflexion) schon immer zur Literaturkritik ${ }^{21}$. Wie Thomas Anz herausarbeitet, ist diese Funktion bei Reich-Ranicki besonders ausgeprägt. In seinen Texten finden sich die „rhetorischen Regeln belehrender Argumentation“ ebenso, wie „die Mittel der Affekterregung (movere) und die Künste, das Publikum zu vergnügen (delectare)"22. In diesem Sinn bieten Reich-Ranickis Kritiken jene „Anschaulichkeit und Spannung“, jene „Pointen“ und jene emotional wirksame „Spannungsdramaturgie“ 23 , die er auch von literarischen Texten erwartet.

Dies bemerkt auch Peter von Matt in einem Gespräch, wenn er den Kritiker fragt, ob ihm der ,Aspekt der Kritik als eines öffentlichen Schauspiels" 24 bewusst sei. In seiner Antwort streitet Reich-Ranicki den „Showcharakter" seiner Kritiken nicht ab. Er streicht vielmehr heraus, dass eine Kritik unbedingt ,für das Publikum interessant“, „lesenswert“" und ,aufschlussreich“ 25 sein müsse. Dabei folgt Reich-Ranicki in seinen frühen Rezensionen oft einem bestimmten Muster: Er greift beschreibende Details aus den besprochenen Texten heraus, die stereotyp eingesetzt werden oder kitschig erscheinen, und leitet daraus eine allgemeine Charakterisierung des ganzen Textes ab, wobei in der Pointe oftmals eine überraschende Kenntlichmachung der Schwäche des Textes gelingt. Dies folgt dem Schema der Kontrastkomik, weil gegenüber dem Pathos des kritisierten Romans die Lakonik der Kritik steht. So will ReichRanicki 1969 den Roman Die Schattengrenze von Dieter Wellershof „Es ist zu schlecht, als daß es ignoriert werden könnte“ 26 - als prätentiös und sprachlich affektiert kennzeichnen. Dazu kritisiert er zunächst die „Verdunkelung des Romangeschehens“27 durch einzelne stilistische Fehlgriffe. Diese würden die eigentliche Inhaltslosigkeit überdecken.

20. - M. Reich-Ranicki, Mein Leben, a.a.O., S. 52-53.

21. - Thomas Anz, ,Theorien und Analysen zur Literaturkritik und zur Wertung“, in: Ders./Rainer Baasner, Literaturkritik. Geschichte - Theorie - Praxis, München, C.H.Beck, 2004, S. 194-219.

22. - Thomas Anz, ,Werten und Fühlen. Zur Rationalität und Emotionalität literaturkritischer Kommunikation - am Beispiel von Marcel Reich-Ranicki“", in: Heinrich Kaulen, Christina Gansel (Hrsg.), Literaturkritik heute. Tendenzen - Traditionen Vermittlung, Göttingen, V\&R unipress, 2015, S. 13-26, hier S. 20.

23. - Ebd., S. 21.

24. - M. Reich-Ranicki, Der doppelte Boden, a.a.O., S. 71-72.

25. - Ebd., S. 72.

26. - Marcel Reich-Ranicki, „Feierliche Undeutlichkeiten. Dieter Wellershof: ,Die Schattengrenze“", in: Ders., Lauter Verrisse. Mit einem einleitenden Essay, Frankfurt a.M./ Berlin/ Wien, Ullstein, 1973, S. 132-137, hier S. 132.

27. - Ebd., S. 134. 
Anhand einer genauen Lektüre schließt Reich-Ranicki die deskriptive Ebene (dazu gehören häufige Motive sowie die durch Adjektive und sprachliche Bilder hervorgerufene Stimmung des Textes) mit der Ebene inhaltlicher Bestimmungen zusammen, etwa indem er in der Arbeitsweise eines klassischen Witzes Mehrbedeutungen von Wörtern nutzt:

Eine besonders wichtige Funktion kommt in diesem Roman der Dunkelheit zu. Der Autohändler fühlt sich von „dunklen Wogen“ umgeben und von „,der unveränderlichen Dunkelheit, von der er sich vorstellte, daß er sie in Stücke schnitt, die immer wieder zusammenwuchsen." Ein „dunkles Zurückebben“ wird uns hier geboten und auch „Würfel aus Dunkelheit [...].“ [...] Schließlich: „Die Dunkelheit stand um ihn als ein unaufhörliches Rauschen und Singen.“ So viel Dunkelheit hat es, glaube ich, schon lange nicht mehr in der deutschen Prosa gegeben ${ }^{28}$.

Reich-Ranickis Pointe funktioniert hier so, dass er die „Funktion“ der Dunkelheit nicht nur darin sieht, ein konkretes Motiv darzustellen und das Geschehen und die Figuren des Textes anschaulich zu machen. Vielmehr zieht er die abstrakte Wortbedeutung von „Dunkelheit“ heran. Die stereotyp eingesetzte konkrete Dunkelheit auf der diegetischen Ebene liest der Kritiker also als obscuritas, als Unverständlichkeit des Erzählten an sich. Dabei gilt für ihn a priori, dass der dunkle Text auch ein langweiliger Text ist. Die Unterhaltsamkeit der Kritik dagegen ist nicht nur Selbstzweck, sondern hat eine aufklärerische Funktion (die auch provozierend wirken und dazu führen kann, dass Kritik als hemmender und ,zersetzender' Faktor wahrgenommen wird $\left.{ }^{29}\right)$. In der folgenden Passage aus der Rezension zu Wellershof etwa führt ReichRanicki anhand sprachlicher Unzulänglichkeit die problematische Fantasie des Autors vor:

Ließ sich dieser feierliche Schmus nicht verhindern? ,... endlos in sie eindringend, steigend, während sie ihm entgegensank ..." Vom Eindringen - übrigens: wieso endlos? Das ist doch geprahlt -, vom Ertrinken und Versinken und ähnlichem hören wir allerlei auch bei Wagner, zumal im „Tristan“: das stammt jedoch aus dem 19. Jahrhundert und wird überdies keinem ohne Musik zugemutet ${ }^{30}$.

28. — Ebd., S. 136.

29. - Wie Reich-Ranicki schreibt, bewirkt dieses Selbstverständnis der Kritik auch eine allgemeine „Kritikfeindlichkeit“, die in Deutschland stark hervortritt, und die dazu führt, dass Kritiker ,für üble Querulanten, permanente Spielverderber und ekelhafte Parasiten“ gehalten werden, ja sogar ,in die Nähe zu Verrätern und Volksfeinden“ gerieten. M. Reich-Ranicki, Über Literaturkritik, a.a.O., S. 15-16.

30. - M. Reich-Ranicki, „Feierliche Undeutlichkeiten“, a.a.O., S. 135-136. 
Der Hinweis auf die Verschmelzungs-, letztlich vielleicht auch Vergewaltigungsfantasien bei Richard Wagner, die sich in sprachlich missglückter Form bei Wellershof wiederfinden, zeigen, was ReichRanicki 1967 auch anlässlich von Alfred Anderschs Roman Efraim bemerkt: „daß Kitsch auch eine moralische Kategorie sein kann“ 31 . Der in der Andersch-Kritik explizit ausgesprochene Vorwurf des Philosemitismus, der auf das Skandalon deutscher Schuldabwehr in den 1960er und 70er-Jahren aufmerksam macht, verdeutlicht, vor welchem Hintergrund Reich-Ranicki als jüdischer Kritiker in der Bundesrepublik sein Programm von Kritik und Unterhaltung entwirft. Die rhetorisch und sprachlich sehr genau konzipierte Unterhaltsamkeit der Rezensionen stehen im Dienst einer Sprachkritik, die immer wieder auch als demokratische Kritik von politisch als abwegig erkannten Vorstellungen kenntlich wird. Nur als Randbemerkung und ohne auf einen detaillierten Vergleich eingehen zu können, soll hier festgehalten werde, dass Reich-Ranickis Strategie in der kritischen Tradition der Polemik Heinrich Heines und Karl Kraus' steht ${ }^{32}$. So wie Heine sich als Medienpionier des Feuilletons und Kraus sich der Lesung und der Schallplattenaufnahme bedient hatten und damit ebenfalls Kritik als Unterhaltung und Unterhaltung als Kritik betrieben, ist Reich-Ranicki Teil eines schnell wachsenden medialen Unterhaltungsbetriebs, der sich in den 1980er und 90er-Jahren vor allem im öffentlich-rechtlichen Fernsehen abspielt.

\section{TV-Image}

Die textuellen Unterhaltungs-Effekte verstärken sich noch, wenn Reich-Ranicki sie in der performativen Inszenierung seiner eigenen Figur nutzt. Wer jemals einem Live-Auftritt Reich-Ranickis beiwohnen durfte, vergisst nie mehr die Kombination aus pointierten, brillant eingeübten Sentenzen und witzigen Wendungen, Schlagfertigkeit, Pathos, Mimik, Gestik - und nicht zuletzt den singulären Sprachgestus, sein leicht zu parodierendes, ikonisch gewordenes Markenzeichen. Vielleicht mag es Reich-Ranicki selbst irritiert haben, aber spätestens seit dem Erfolg der TV-Sendung Das Literarische Quartett (ZDF, 1988-2001) ist er über seine Person hinaus zu einer medial vervielfachten Figur der populären Unterhaltung geworden, zu einem quasi-mythologischen Objekt der populären Kultur.

31. - Marcel Reich-Ranicki, „Sentimentalität und Gewissensbisse. Alfred Andersch: ,Efraim““, in: Ders., Lauter Verrisse, a.a.O., S. 39-46, hier S. 45.

32. - Vgl. Andreas Stuhlmann, ,Die Literatur - das sind wir und unsere Feinde“. Literarische Polemik bei Heinrich Heine und Karl Kraus, Würzburg, Königshausen \& Neumann, 2010. 
Diese Ikone hat sich von den eigentlichen Inhalten (man könnte diese als Reich-Ranickis tatsächlich immenses Wissen über Literatur, seine trotz allem oft enorm treffsicheren ästhetischen Urteile verstehen) abgelöst und besteht in der medialen Sphäre fast nur noch aus dem Image des Kritikers mit dem rollenden R. Als Reich-Ranicki 1996 zum ersten Mal in Die Harald Schmidt Show auftritt, kündigt ihn Schmidt mit den Worten an: „Ein Mann, der viele, viele Titel hat. Literaturpapst ist der häufige, den man in Verbindung mit ihm hört. Ich würde sagen, er ist ein großartiger Entertainer!“ Reich-Ranicki akzeptiert diesen Titel breitwillig. Unter dem Beifall und Gelächter des Publikums, sagt er im Gespräch, dass ihm zunächst vorgeworfen wurde, ein "Schulmeister“ zu sein, dann ein „Entertainer“. Und: „Genau das möchte ich sein: Schulmeister und Entertainer in einem." Neben der Pädagogik wolle er explizit auch „Unterhaltung“ bieten, wobei er nicht vergisst zu erwähnen, dass der Begriff Unterhaltung, ,in Deutschland ein böses Wort“" sei ${ }^{33}$.

Allerdings kann die "Tendenz hin zur Entgrenzung von U.[nterhaltung] “34 natürlich auch im deutschsprachigen Raum gegen Ende des 20. Jahrhunderts beobachtet werden. Bezeichnenderweise nennt das Historische Wörterbuch der Rhetorik unter dem Lemma „Unterhaltung“ zur Veranschaulichung dieser Tendenz gerade Die Harald Schmidt Show, die stark mit Selbstreferentialität arbeitet. Die Literaturkritik ist also mit Reich-Ranicki vollkommen in der Unterhaltung angekommen, die Unterhaltungsfunktion ist hypertroph geworden und dominiert alle anderen Funktionen der Kritik. Umgekehrt kann wiederum auch hier beobachtet werden, dass sich aus der Unterhaltsamkeit Momente ergeben, die sich in ihrer Ambiguität einer einfachen Deutung entziehen.

In der Live-Sendung Das Literarische Quartett war Unterhaltung bekanntlich Programm. Es ist banal, wenn festgestellt wird: „Der Unterhaltungswert der Sendung begründete sich dabei eher auf der partnerorientierten als auf der problemorientierten Gesprächsebene" 35 . Jede Zuschauerin und jeder Zuschauer musste schnell merken, dass der Showcharakter der Sendung erheblich auf dem polemisierenden, provozierenden und profilierenden Rollenspiel und den Rollenzuweisungen der Diskutierenden beruht. Diese führen wie Akteure in einer Sitcom ihre Kontroversen nach dem Muster von Running Gags.

33. - Vgl. den Auftritt auf youtube.

34. - T. Pekar, „Unterhaltung“, a.a.O., Sp. 928.

35. - Rainer Hartmann: Literaturkritik im literaturfernen Medium Fernsehen. Literaturvermittlung im Spannungsfeld zwischen kritischem Anspruch und TV-Realität am Beispiel des ,Literarischen Quartetts" mit Marcel Reich-Ranicki, Marburg, Verlag LiteraturWissenschaft, 2011, S. 17. 
Die Sitcom lässt sich auch als fortlaufender, subversiver Kommentar $\mathrm{zu}$ den heute oftmals vergessenen, schwerfüßigen, absolut humorlosen Texten der deutschsprachigen Literatur verstehen. So wurde beispielsweise in der siebten Folge vom 12. Oktober 1989 Botho Strauß' Buch Fragmente der Undeutlichkeit erwähnt. Im Unterschied zum in der gleichen Folge vorher diskutierten Essay von Peter Handke, den Sigrid Löffler ausgesprochen positiv und Reich-Ranicki ausgesprochen negativ beurteilt, findet sich die Runde bei Strauß in der negativen Reaktion einig (Strauß sei, so Löffler, ,ein verschwiemelter Mystagoge“, ein Urteil, das in Hinsicht auf Strauß' spätere kulturkonservative und rechtsnationale Äußerungen hellsichtig scheint). Als Reich-Ranicki das Büchlein präsentiert, spricht er ausschließlich über den Verlag und die an Strauß verliehenen Preise. Über den Inhalt, also über Strauß' Text zum amerikanischen Dichter Robinson Jeffers und über die im zweiten Teil gesammelten Aphorismen, wird nichts gesagt: „Er hat ein neues Buch veröffentlicht. [...] Ja, ja [gespielt nachdenklich] ... wer hat denn das gelesen?“

Zunächst ist Reich-Ranicki mit dieser rhetorischen Frage das Gelächter des Publikums sicher. Aber sie ist auch ein dramaturgischer Kniff, um das negative Urteil über den Text bereits vor dem Gespräch festzulegen. Wenn Strauß mit der Devise „Zurück ins Nicht-Verstehen!“36 die Literatur als moralisch indifferente, irrationale Urmacht umschreibt und dabei so bierernst wie nur irgendwie möglich vorgeht, so ist dies das genaue Gegenteil von Reich-Ranickis Ideal. Dieser spielt deshalb auf unterhaltsame Weise eine Figur, die ironisch die Nicht-Unterhaltsamkeit und damit eben auch die intellektuelle Bedenklichkeit des besprochenen Textes kenntlich macht. Dieser Spielcharakter lässt den unterhaltungs- oder konsumkritischen Vorwurf ins Leere laufen, „,er Anspruch auf Authentizität“ 37 würde im Literarischen Quartett verraten. Denn vor dem Hintergrund einer differenzierten Konsumästhetik ist klar, dass eine Talkshow niemals Echtheit im Sinne eines tief empfundenen Gefühlsbezugs zu den Texten bietet - eine Echtheit, die notabene unter den Bedingungen der Moderne immer schon ein Phantasma darstellt, das tief mit reaktionären Weltbildern verbunden ist.

Demgegenüber führt die Show ein Spiel mit Echtheitsvorstellungen vor, das sich aber nicht im Selbstzweck erschöpft. Der „Anspruch“ auf vermeintlich authentische Äußerungen wird von Anfang an immer nur ironisch verstanden und damit unterlaufen, was wiederum die kritische Funktion der Unterhaltung stärkt. Im Fall der Fragmente der

36. - Botho Strauß, Fragmente der Undeutlichkeit, München, Carl Hanser, 1989, S. 50.

37. - Nicht folgen kann ich deshalb den entsprechenden Ausführungen bei J. Ahmadi, Der Papst und der Bienenkorb, a.a.O., S. 214. 
Undeutlichkeit zielt die Kontrastkomik Reich-Ranickis auf das antiquierte Pathos von Strauß' neoromantischer Authentizitäts-Emphase, die das poetische Wort ${ }^{38}$ in pseudo-heidegger'scher Manier als Gegenwelt zu Technik und liberaler Demokratie ${ }^{39}$ stilisiert.

Dies zeigt sich noch stärker im folgenden Gespräch, in dem die Diskutierenden auf die vorhergehende Kontroverse über Handke zurückkommen. Löffler sagt, dass Reich-Ranicki sie schon am Telefon über seine negative Meinung informiert hätte. Reich-Ranicki nimmt den Faden nun wieder auf:

Ich werde hier nicht zitieren, was Sie mir am Telefon gesagt haben [Winken mit der rechten Hand]. [...] Was Sie mir über dieses Buch von Handke gesagt haben, werde ich nicht zitieren. [laut werdend] Dazu bin i... nein, nein, nein, nein, dazu bringen Sie mich nicht! [Löffler flehend: Tun Sie's doch!] ... nein, nein, nein, n...n... provozieren Sie mich nicht! [Pause, dann mit Blick über den Brillenrand im gedämpften Ton:] Wollen Sie's wissen?

Das kleine, klamaukartige Stück, das Reich-Ranicki an dieser Stelle inszeniert - unterstützt von Sidekick Löffler -, ist genau getaktet. Die Pointe ist präzise gesetzt. Mit Kritik im intellektuellen Sinn hat die Passage nichts zu tun, es dominiert offensichtlich die Unterhaltungsfunktion. Aber wie funktioniert diese Unterhaltsamkeit?

Zunächst spielt Marcel Reich-Ranicki mit seinem Image als allmächtiger Kritiker, dessen Meinungen von großem Gewicht sind. Die damit kontrastierende Leichtigkeit ergibt sich aus seinem performativ vorgezeigten Narzissmus, der freilich nicht negiert, sondern verdoppelt wird. Diese Meta-Selbstironie hebt sich wiederum deutlich ab von den eigentlich besprochenen Texten. Wenn dort vom „Ungrund des Morgens" 40 oder vom „Gelaß des Selbst" ${ }^{“ 41}$ die Rede ist, dann ist dieser Text eben gerade nicht ,einschweigbar“ (S. 48), sondern gegen seine eigene Behauptung narzisstisch, prätentiös und banal. „Philosophische Banalität entsteht" aber, so bemerkt Theodor W. Adorno, mit dessen Begriff von Kritik Reich-Ranicki sich einig weiß, „wo dem allgemeinen Begriff jene magische Teilhabe am Absoluten zugeschrieben wird, die

38. — , ,...] der Poet, der Ergänzer der technischen Metapher, von dieser selbst auf den Plan gerufen, um sie zu brechen, zu öffnen, wieder einschweigbar zu machen und den Geist vor eine abrupte, unergründliche Schönheit zurückzuführen -“. B. Strauß, Fragmente der Undeutlichkeit, a.a.O., S. 48.

39. — „Im Herzen schlummert der König, der wache Verstand wacht über die Demokratie, der Geist ist Theokrat und die Phantasie schließlich sucht nach der labyrinthischen Ordnung totalitärer Zentralen." Ebd. S. 57. Die Passage ist natürlich deutungsbedürftig, allerdings vielleicht auch gar nicht weiter deutbar.

40. - Ebd., S. 59.

41. — Ebd., S. 49. 
seine eigene Begrifflichkeit Lügen straft" “42. Tatsächlich stellt Strauß’ archaisierende Unmittelbarkeitsromantik noch so etwas wie „metaphysische Winterhilfe in Permanenz" 43 dar, wie Adorno sarkastisch den auf Heidegger zurückgehenden ,Jargon der Eigentlichkeit' mit dem nationalsozialistischen Begriff der „Winterhilfe“ charakterisiert. Die Unterhaltung, die von Reich-Ranickis Inszenierung ausgeht, ist ein Antidot gegen dieses Geschwollene: Ihre Komik ist völlig uneigentlich, insofern sie explizit tendenziös und damit ernüchternd ist. Sie ist oberflächlich simpel, doch sehr genau komponiert. Sie erscheint zunächst selbstzentriert, arbeitet aber mit mehreren Ironie-Dimensionen. Das ist Unterhaltung, aber es ist auch Kritik.

\section{Holocaust und Mediengesellschaft}

Mit seiner enorm erfolgreichen Autobiografie wird die Ikone Marcel Reich-Ranicki in der breiten öffentlichen Wahrnehmung um einen Aspekt erweitert: Seinen Status als Holocaust-Überlebender. Auch wenn die entsprechenden Episoden in Mein Leben nur wenige Teile des Textes ausmachen, sind sie für den Erfolg des Buches wohl ausschlaggebend. Die Verschiebung des Images vom ebenso wortgewaltigen wie unbarmherzigen Kritiker zum Zeitzeugen, vom Mächtigen zum offensichtlich Ohnmächtigen, der ,zufällig verschont“"44 wurde, während seine Familie umgebracht wurde, der sein Leben lang Angst hat ${ }^{45}$, findet im Auftritt des greisen und zerbrechlich wirkenden ReichRanickis im deutschen Bundestag anlässlich der „Gedenkstunde für die Opfer des Nationalsozialismus“ am 27. Januar 2012 seinen definitiven Ausdruck. Dieser Auftritt wurde vom Seminar für allgemeine Rhetorik der Universität Tübingen als „Rede des Jahres“ ausgezeichnet. Als Begründung wurde genannt, dass sich der Redner „der konventionellen Gedenkrhetorik" verweigere, zudem verzichte er „auf Appelle, Mahnungen oder Forderungen. Stattdessen rückt er das Prinzip der Evidenz, namentlich die Vergegenwärtigung eines entscheidenden Moments in der Vernichtungsgeschichte der Juden, in den

42. - Theodor W. Adorno, Jargon der Eigentlichkeit. Zur deutschen Ideologie, in: Ders., Gesammelte Schriften, hrsg. v. Rolf Tiedemann unter Mitwirkung von Gretel Adorno, Susan Buck-Morss und Klaus Schultz, Bd. 6, Frankfurt a. M., Suhrkamp, 2003, S. 413-526, hier S. 447.

43. - Ebd., S. 463.

44. - M. Reich-Ranicki, Mein Leben, a.a.O., S. 303.

45. - In einem Interview anlässlich der Verfilmung seiner Autobiografie äußert sich Reich-Ranicki: „Die Angst vor der deutschen Barbarei [...] hat mich ein Leben lang begleitet.“ Im Gespräch: Marcel Reich-Ranicki. „Es gibt keinen Tag, an dem ich nicht an das Ghetto denke", Frankfurter Allgemeine Zeitung, 09.04.2009, https://www.faz. net/aktuell/feuilleton/im-gespraech-marcel-reich-ranicki-es-gibt-keinen-tag-an-demich-nicht-ans-getto-denke-1783896.html (Zugriff am 05.06.2019). 
Vordergrund“"46. Bereits Reich-Ranickis Autobiografie ist nicht politisch im Sinne eines didaktischen Ziels einer Aufarbeitung des Geschehenen. Gemäß einer Äußerung, die er 2001 bezeichnenderweise in der Talkshow des Moderators Michel Friedman tätigte, sei es seine Absicht gewesen, $\mathrm{zu}$,beschreiben, was ich erlebt habe“. Es sei „nicht die Aufgabe der Juden in diesem Land, das deutsche Volk politisch zu erziehen.“

Entgegen seiner vorher jahrzehntelang verfolgten Intention, ein „Schulmeister“ der Literatur zu sein, hegt Reich-Ranicki diesen Anspruch im politischen Bereich also nicht. Doch gerade die gerühmte „Evidenz" seiner Schilderungen von Massenmord und Überleben - „eine kunstvolle und ergreifende, aber an keiner Stelle pathetische Erzählung" 47 - führt paradoxerweise auch zu ihrer Unterhaltsamkeit. Reich-Ranickis Vortrag wirkt zunächst kunstlos, ist aber rhetorisch tatsächlich äußerst kraftvoll konstruiert. Vor allem der Schlusssatz mit dem parataktischen Aufschub und der tautologischen Wiederholung „Ziel - Zweck“, der im Wort „Tod“ kulminiert, zeigt seine rhetorische Meisterschaft: „Die Aussiedlung aus Warschau, sie hatte nur ein Ziel, sie hatte nur einen Zweck, den Tod. - Ich danke Ihnen." Es wurde bisher kaum bemerkt, dass Reich-Ranicki auch als erzählender Zeitzeuge nicht aufhört, ein Kritiker und Sprachkritiker zu sein. Dieser letzte Satz der Rede demonstriert schlüssig, wie die deutschen Verantwortlichen das Verbrechen auch sprachlich, mit Lügen und Phrasen, ermöglichten. So macht Reich-Ranicki mit präzisen Mitteln den banalen Begriff der „Aussiedlung“ als Hohlraum des Terrors kenntlich.

Mein Leben zeigt Reich-Ranicki als Figur verschiedener historischer Welten: der Weimarer Republik, des Warschauer Gettos, des kommunistischen Polens und schließlich der alten Bundesrepublik, die erzählerisch am meisten Platz einnimmt. Darin schildert Reich-Ranicki auch einige wichtigen Stationen des deutschen Verhältnisses zum Holocaust und zum Judentum: Die Kontroversen um Rainer Werner Fassbinders Stück Der Müll, die Stadt und der Tod (1974), den Historikerstreit (1986) und die skandalösen Auswirkungen von Martin Walsers Rede in der Frankfurter Paulskirche 1998. Reich-Ranickis rückblickende Einschätzungen dieser Debatten macht klar, dass sein Leben längst auch durch die Berliner Republik geprägt worden ist beziehungsweise, dass er diese prägte. Um das Jahr 2000 verschiebt sich die Bedeutung des Holocausts und damit auch der Begriffe ,Judentum“ und „Jude“ in der deutschen Öffentlichkeit, was sich prominent in den jahrelangen Kontroversen zum 2005 in BerlinMitte eingeweihten Denkmal für die ermordeten Juden Europas niederschlägt. Von einem Thema an den Rändern des historischen Bewusstseins

46. — http://www.rhetorik.uni-tuebingen.de/portfolio/rede-des-jahres/ (Zugriff am 05.06.2019).

47. - Ebd. 
- von dem man nur in Anspielungen oder gar nicht spricht 48 - wandert der Holocaust nicht nur im übertragenen Sinn ins Zentrum der neuen Republik. Der Holocaust erlangt eine neue Sichtbarkeit. Als ,Zivilisationsbruch“ein Begriff, den Dan Diner 1989 in den Sprachgebrauch eingeführt hat 49 - bildet er nun den dunklen Grund des deutschen Selbstbildes, dem man sich politisch immer wieder zu versichern hat. Damit werden auch jüdische Figuren der Kultur und des Zeitgeschehens viel stärker als „Juden“ wahrgenommen, eine freilich eher diffuse Kategorie des Opfers deutscher Gewaltgeschichte.

Der ,jüdische“ Reich-Ranicki wird nun also vermehrt auch in der Öffentlichkeit als Repräsentant einer jüdischen Geschichte identifiziert. So verfassen beispielsweise Schülerinnen und Schüler 2008/09 an einem Gymnasium für eine Webseite zu einem ,Wahlgrundkurs Jüdische Geschichte und Kultur" auch einen biografischen Eintrag zu Marcel Reich-Ranicki ${ }^{50}$, was noch fünfzehn Jahre vorher kaum selbstverständlich gewesen wäre. Doch diese Wahrnehmungsverschiebung, die auch mit einer Historisierung der deutsch-jüdischen Diskurse in der alten Bundesrepublik einhergeht, findet parallel $\mathrm{zu}$ einer Verschiebung der Deutungsfelder des Holocausts statt. Bis zum Ende des 20. Jahrhunderts blieb das Thema des Holocausts größtenteils einer akademischen Beschäftigung und der Beschäftigung in der Hochkultur vorbehalten, in der freilich die ,,herkömmlichen Narrationen und Repräsentationsweisen" 51 erprobt und zerschlagen werden. Auseinandersetzungen mit Holocaust und Zweiten Weltkrieg erfahren seit den 2000er-Jahren ,,verstärkt eine Ausdehnung auf Neue Medien und globale Kontexte", nach einem Primat des Authentischen und der Zeugenschaft spielen zudem experimentelle Verfahren,,in den Künsten ebenso wie in der Popkultur und der Gedenkkultur" ${ }^{652}$ eine große Rolle.

Der Holocaust ist schon seit Jahrzehnten ein Teil der Popkultur und der medialen Unterhaltung, stets begleitet von Kontroversen und

48. - Als Beispiel seien etwa Hans Werner Richters in seinem Buch Im Etablissement der Schmetterlinge (1986) enthaltene literarische Porträts jüdischer Autoren der Gruppe 47 genannt, ohne die Worte ,Jude“ oder ,jüdisch“ zu nennen, obwohl es sich dabei - wie Reich-Ranicki schreibt - um Autoren handelt, „deren Persönlichkeit und deren literarisches Werk von der Zugehörigkeit zur jüdischen Minderheit und von der Vertreibung aus Deutschland in hohem, in höchstem Maße bestimmt wurden.“ M. Reich-Ranicki, Mein Leben, a.a.O., S. 411.

49. - Dan Diner, ,Vorwort des Herausgebers“, in: Ders. (Hrsg.), Zivilisationsbruch. Denken nach Auschwitz, Frankfurt a.M., Fischer Taschenbuch, 1989, S. 7-14.

50. — http://www.judentum-projekt.de/persoenlichkeiten/liter/reichranicki/index. html (Zugriff am 04.08.2019).

51. - Esther Kilchman, „Zum Band“, in: Ders. (Hrsg.), Artefakte. Holocaust und Zweiter Weltkrieg in experimentellen Darstellungsformen in Literatur und Kunst, Köln, Weimar, Wien, Böhlau, 2016, S. 7-18, hier S. 8.

52. — Ebd., S. 9. 
Debatten zur moralischen Angemessenheit solcher Repräsentationen ${ }^{53}$. Dies beginnt spätestens mit der amerikanischen Miniserie Holocaust (1978), führt über die Rezeption von Steven Spielbergs Schindler's List (1993) bis hin zu Repräsentationen auf youtube oder zum neusten Video der Band Rammstein und ihres Songs „Deutschland“ (2019). Statt stilles Gedenken und Darstellung stehen in der Popkultur der Event und die digitale Medialität im Vordergrund, so ,werden gegenwärtig auch die Karten der Holocaust-Repräsentation und ihrer Akzeptanz neu gemischt" "54. Im Zuge des Generationenwandels und der veränderten Wahrnehmung der Konsumkultur sowie aufgrund der Digitalisierung transformiert sich die Vergegenwärtigung des Historischen. Kirstin Frieden hält in einer Studie zu Neuverhandlungen des Holocausts fest: ,Nicht die historische Erfahrung, sondern Erlebnisse eines gegenwärtigen Lebensgefühls fingieren neue Deutungs- und Identifikationsangebote, welche vor allem durch die Medien, Werbung und subkulturelle Merkmale bestimmt sind“"55.

Reich-Ranicki erfüllt diese Voraussetzungen der Mediengesellschaft nahezu perfekt. Als bereits populäre, ikonische Medienfigur bedient er die Mechanismen einer Aufmerksamkeitsökonomie der Knappheit und der Erregungserzeugung. Dabei ist er aber immer noch Zeitzeuge. In dieser doppelten Identität transformiert sich in seinem Image das historische Gedächtnis, das auf Authentizität und Aufmerksamkeit beruht, in eine parahistorische Repräsentation, die sich an Medialität ausrichtet.

Diese Transformation verdichtet sich in der Verfilmung der Autobiografie unter dem Titel Marcel Reich-Ranicki: Mein Leben durch den Regisseur Dror Zahavi und den Drehbuchautor Michael Gutmann 2009 für die ARD. Der im Ganzen recht nüchtern erzählte Fernsehfilm ist mit seinem eingängigen Narrativ unterhaltsam und will auch unterhalten, womit er sich von intellektuell herausfordernden filmischen Annäherungen wie Claude Lanzmanns Film Shoah (1985), der sich jeglicher fiktionalen Darstellung verweigert, oder László Nemes' gerade auf provozierende Fiktionalität setzendem Film Sauls Sohn (2015) unterscheidet. Als Figur der Filmhandlung ist der Überlebende Marcel Reich-Ranicki vollkommen in der Unterhaltung aufgehoben. Doch entfaltet sich durch diese Unterhaltsamkeit auch seine Wirkung als Kritiker von falschem Pathos und Sentimentalität. In der Performance Marcel Reich-Ranickis wird die Kritik also ganz zur Unterhaltung, aber die Unterhaltung erreicht auf verschiedenen Ebenen eine kritische Funktion, die sich ohne Didaktik in den Dienst der liberalen und offenen Gesellschaft stellt.

53. - Vgl. Caspar Battegay, Judentum und Popkultur. Ein Essay, Bielefeld, transcript Verlag, 2012, S. 91-115.

54. - Kirstin Frieden, Neuverhandlungen des Holocaust. Mediale Transformationen des Gedächtnisparadigmas, Bielefeld, transcript Verlag, 2014, S. 24. 55. - Ebd. 
